\title{
Means of reducing pain in farm animals
}

\author{
O.L. Levionnois ${ }^{1}$ and P. Mormède ${ }^{2}$ \\ ${ }^{1}$ Section for Anaesthesia and Pain Therapy, Department of Clinical Veterinary Sciences, Vetsuisse Faculty, University of Bern, Switzerland (Länggassstrasse, \\ 124 CH-3012 BERN); ${ }^{2}$ INRA, UMR1388, Génétique, Physiologie et Systèmes d'Elevage, F-31326 Castanet-Tolosan, France
}

\section{Means of reducing pain in farm animals}

The aim of this article was to identify avenues for eliminating or reducing pain in animals while also taking into account their practicality and cost. The principles and means of action are first described in general terms, and then examined in detail for several procedures that have been identified as being painful, such as castration or slaughter. Possible actions based on new genetic selection models are also envisaged.

\subsection{General approach for reducing pain on farms}

Article entitled, Sources of known and/or potential pain in farm animals, pointed to both the reality and the complexity of the issue on pain in farm animals as it is raised in livestock production but also in human health and safety and cultural considerations.

The welfare of livestock in intensive farming systems is based on five basic rights, as defined by the experts in the Brambell Committee in 1965. One of those rights explicitly mentions the absence of pain, injuries and diseases, by preventive veterinary care, rapid diagnosis and appropriate treatment, as a condition for the welfare of animals.

5.1.1. Major principles. The use of laboratory animals for scientific research has been the topic of extensive discussions aimed at minimising and better justifying the resort to in vivo experimentation. This deliberation led to a methodological problem-solving approach aimed at providing guidelines for the use of animals in experiments. This approach proves to be exemplary in achieving one of the major goals of the present expert assessment: structuring pain management and reducing pain in farm animals. The principles of the 3Rs (replace, reduce, refine) have been widely accepted since the 1960s and now strongly regulate the use of animals in experimental research. We will refer to them in the consideration of alternatives that eliminate (suppress) sources of pain in farm animals. The second step is to 'substitute' painful procedures with others that are less painful. Most often this involves seeking techniques for which the intensity or impact of pain is minimised. If it is not possible to eliminate (suppress) pain completely, consideration should be given to relieving it (soothing) with pharmacological treatments such as analgesia or the use of local anaesthetics. The analogy with the '3Rs', designed to reduce the number of animals used in experimental procedures, led us to follow a similar methodology to limit pain in farm animals based on the '3Ss': eliminate sources of pain (suppress), substitute painful procedures by other means (substitute), and relieve pain in farm animals (soothe).

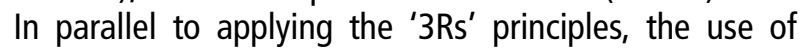
laboratory animals has been monitored by establishing local ethics committees. The role of these committees is to supervise the use of animals in research and to assess whether the integrity of the laboratory animals is maintained. Although it would be more difficult to implement, it seems obvious that a similar surveillance of farming practices would permit a better understanding of and control over the sources of pain associated with the production of food derived from animals.

Overall, solutions found in the literature to improve upon the use of procedures known to be painful can be divided into five categories: (1) if the procedure is not justified and in the absence of alternatives, stop using the procedure (eliminate the source of pain: suppress), (2) whenever possible, rear animals that no longer require the use of such procedures (eliminate the use of sensitive animals: suppress), (3) replace the procedure with another one that is less painful (substitute), (4) improve the procedure in order to limit pain intensity (substitute) and (5) relieve pain (soothe). Proposing the ultimate solution, which is to stop livestock production and therefore to stop eating animal products such as meat (vegetarianism) or even eggs and milk (veganism), does not fall within the scope of this assessment. Hence, this article focuses on farm practices causing pain, the sources of which are presented in the previous article, and the possible improvements that may be made on them.

5.1.2. The means of action. Several means are available to mitigate the suffering of farm animals:

1. focusing on animals: changing genetic selection processes by including new criteria along with performance checks,

2. focusing on industry conditions: changing farming practices and/or rearing conditions, changing the laws, training farmers, encouraging rearing requirements that take less 
painful practices into consideration, improved problem management,

3. focusing on pain management: use of anaesthesia or analgesic drugs.

Focusing on animals: genetic improvement. The main focus of selection criteria for farm animals over the last 40 years has been on increasing productivity and improving product quality, such as selecting for leaner meat. This type of genetic selection has probably favoured the emergence of fear behaviour, abnormal aggressive behaviour or a reduced ability to adapt to environmental constraints (e.g. resistance to heat), the development of musculoskeletal disorders, birthing difficulties (cattle), reduced survival of newborns in favour of higher fertility (pigs), or increased susceptibility to diseases. Robustness (or 'functional' traits) has been taken into account in selection schemes since the late 1990s. For example, this applies for the leg conformation (poultry and pigs), the ease of calving (animals) and the newborn survival rate (pigs), the number of somatic cells in milk (dairy cows). This strategy has shown its effectiveness in reducing some sources of pain (less lameness, improved neonatal survival, decreased infection rates) and should be continued or even extended to other more complex characters (general resistance to stress, selecting against behavioural disorders). It should be noted, however, that the time required for the impact of such strategies to be visible depends on several factors: the heritability of the character, the selection pressure or the selection schemes implemented, as well as the interval between generations. Hence the benefit is only perceptible after several years.

Finally, since most painful situations are of multifactorial origin and include the effects of housing and animal management, genetic selection by itself is not sufficient to eliminate the problems.

Focusing on industry conditions. According to the context there is currently some leeway for minimising or eliminating certain sources of pain caused by the industry conditions. This would mainly involve improving the facilities and the techniques or practices in use on farms and in slaughterhouses. Attempts to improve the welfare of farm animals are focused on three main factors that are often inter-linked: (1) the physical health and the satisfaction of the physiological needs of animals, (2) the minimisation of negative situations (pain, fear, anxiety) and (3) the ability for animals to express their natural behaviours.

In the livestock industry increases in production efficiency need to be balanced with maintaining consumer safety from health risks (e.g. ensuring the absence of drug residues and diseases). Thus, the means proposed to minimise or eliminate pain in the specific context of a livestock production system must take into account the legal, economic, health and safety and cultural constraints for farmers, and food safety constraints for consumers, as well as the medical and behavioural constraints for the animals.

Focusing on pain management. Pain is treated by administering drugs that affect either the ability to feel pain (general anaesthetics administered intravenously, intramuscularly or by inhalation) or the physiological mechanisms of pain (painkillers or analgesics). Anaesthetics induce a loss of consciousness, and inhibit memory and the unpleasant perception of a noxious stimulus, although there are still changes in physiological markers. Painkillers or analgesics, administered orally or intramuscularly, reduce the animal's sensitivity to pain. Non-allopathic analgesic treatments (homeopathy, naturopathy) and non-pharmacological treatments (osteopathy, acupuncture, physical restraining techniques such as the twitch for horses or hypnotic postures for sheep) are also available. However, there is insufficient scientific data on their effectiveness and their usefulness in specifically reducing pain to promote their use on a wide scale. It is nonetheless particularly worthwhile considering these alternatives in the context of farming since they reduce the need to resort to expensive allopathic treatments that are sources of residues in animal products.

\subsection{Alternative means for preventing or reducing pain in farm animals}

\subsection{1. 'Suppress': can sources of pain in livestock be eliminated?}

Non-surgical as opposed to surgical castration. Whatever the species or the method, castration of males is considered to be painful when performed without anaesthesia or analgesia (see pages 319-332). For cattle and poultry, there is no current alternative to surgical castration. In pigs, this procedure can be replaced by rearing intact males or by immunocastration (Table 1) as already practiced in some countries (e.g. intact males in England and Spain, immunocastration in Australia and Brazil).

However, all of these alternatives present disadvantages in their implementation (Table 1). In countries where piglets are not castrated, consumers complain about the presence of malodorous compounds in the meat. These are mainly androstenone, a pheromone secreted by the testicles, and skatole, a molecule derived from the intestinal metabolism of tryptophan, of which the degradation in the liver is inhibited by sex hormones. Pain relief (soothing) during surgical castration with anaesthesia and analgesia will be discussed later (see Section 5.3.3.).

Rearing entire male pigs for consumption is feasible if the percentage of animals with undesirable odours is reduced to a level that is economically acceptable, and if a reliable method of odour assessment can be put in use on the slaughter line. For effective evaluation of these odours, thresholds of acceptability for consumers must be defined and effective and inexpensive methods to quantify odours must also be available. Some procedures have been evaluated or are under development, including the detection of board taint by human nose. Genetic factors play an important role in controlling the amount of androstenone in the fat, and to a lesser extent, that of skatole which is more dependent on nutritional and environmental conditions. 
Table 1 Advantages and disadvantages of possible methods as alternatives to surgical castration of piglets without anaesthesia

\begin{tabular}{|c|c|c|}
\hline Alternative method & Advantages & Disadvantages \\
\hline $\begin{array}{l}\text { (1) Rearing entire males in association with } \\
\text { (a) Sorting carcasses to remove those } \\
\text { with too strong an odour } \\
\text { (b) Genetic selection to limit the release of } \\
\text { malodorous substances }\end{array}$ & $\begin{array}{l}\text { - Leaner carcasses (better nutritional } \\
\text { value of meat) } \\
\text { - Reduced nitrogen excretion due to } \\
\text { better protein retention, hence less } \\
\text { nitrogen in effluent } \\
\text { - Lower production costs due to better } \\
\text { efficiency of food conversion }\end{array}$ & $\begin{array}{l}\text { - Presence of sexual odours due to the presence of } \\
\text { androstenone and skatole } \\
\text { - Other meat quality problems: 'dark cutting' and/ } \\
\text { or less tender meat and softer fat } \\
\text { - More aggression and mounting behaviour, and } \\
\text { therefore deterioration of welfare conditions for } \\
\text { some animals }\end{array}$ \\
\hline (2) Immunocastration & $\begin{array}{l}\text { - Efficient method to remove the sexual } \\
\text { odours associated with the presence of } \\
\text { androstenone and skatole } \\
\text { - Feed conversion efficiency and nitro- } \\
\text { gen excretion prior to immunisation } \\
\text { close to that for entire males } \\
\text { - Behaviour close to that of castrated } \\
\text { males once they are immunised (less } \\
\text { aggressive behaviour and mounting) } \\
\text { - Available: vaccine received the Eur- } \\
\text { opean marketing authorisation in } 2009\end{array}$ & $\begin{array}{l}\text { - Inspection of carcasses required to verify the } \\
\text { effectiveness of the immunisation } \\
\text { - Behaviour close to that of entire males (mounting } \\
\text { and aggression) prior to immunisation } \\
\text { - Reluctance of consumers because of vaccination } \\
\text { against a sex hormone }\end{array}$ \\
\hline $\begin{array}{l}\text { (3) Surgical castration with anaesthesia } \\
\text { and analgesia }\end{array}$ & - No side effects & $\begin{array}{l}\text { - Additional costs compared with current practices } \\
\text { - The administration of analgesia and anaesthesia } \\
\text { only by veterinary surgeons or also by other } \\
\text { authorised persons is currently being debated }\end{array}$ \\
\hline
\end{tabular}

The identification of the genes involved in the production of androstenone and skatole has given some hope for a solution, or at least a partial solution, through genetic selection. However, the task is complicated by potential negative effects of a selection against androstenone on sexual maturation and the performance of the animals.

Immunocastration is another possibility and a commercial vaccine currently in use in some countries (e.g. Australia and Brazil) received European marketing authorisation (MA) in 2009. Although the method has been proven successful on a technical level, some animals may be missed in the vaccination programme and therefore inspections will be required to ensure the carcasses are free of sexual odours (with checking procedures similar to those described previously for entire male carcasses). Furthermore, the effects on animal welfare are still poorly documented. Other uncertainties lie in the acceptability of the method to the general public and the risks taken by the personal handling and administering the vaccine. A recent Swedish survey shows that the potential health risk associated with the use of biotechnology is acceptable to Swedish consumers in order to improve animal welfare, so long as the taste of the meat is unchanged. In Switzerland, castration using anaesthesia and analgesia is being maintained in parallel to immunocastration so as both categories of products can be offered to consumers.

In conclusion, surgical castration of piglets without anaesthesia or analgesia could be replaced by immunocastration and the production of entire male pigs. Neither of these solutions is completely satisfactory at the present time and their implementation requires an adjustment of the processing of carcasses by the pig industry.

The case of castration of broilers (caponisation). Caponisation or castration of chickens is mainly practiced in slow growing breeds. The primary objective is to obtain meat with specific organoleptic qualities which requires that the rooster does not reach sexual maturity. Another advantage of caponisation is that it prevents the expression of aggressive behaviour, including pecking and cannibalism, which is most often seen in sexually mature animals. It should be noted that there are currently no satisfactory alternatives to castration without anaesthesia or analgesia (see Section 5.3.3). Caponisation has been discontinued in some countries, including Belgium.

The case of teeth clipping in piglets. Stopping the practice of teeth clipping could be envisaged without causing major problems. Some studies have shown that giving up this practice did not result in more damage to the teats of the sows although there was a slight increase in skin lesions in piglets. As stated in French and European regulations, teeth should only be clipped in specific cases when injuries are seen in sows or piglets and when other possible causes (lack or insufficient production of milk) have been excluded. Teeth clipping is excluded in organic farming (see pages 319-332) with promising results but the rearing conditions differ to most farming standards on a health and structural basis. There is very little data from long-term studies available to help conclude on the possibility of extending such measures 
to a larger scale. The presence of skin wounds that piglets inflict on each other, however, has to be taken into account. Establishing a special code of practice without teeth clipping could incite farmers to implement these measures if associated with consumer approbation. For consumer recognition to be an effective encouragement it is essential to have good communication on the objectives (health, nutritional or organoleptic characteristics) and advantages (animal husbandry, welfare) of the different production systems, labelling and classification of products that are on offer.

\section{Alternatives to tail docking in various farming systems}

The case of tail docking in cattle. Tail docking in dairy cattle is an interesting case of a painful practice that was used in past years and was given up without any repercussions on health or economic impact. Until recently, docking was mainly used to reduce the incidence of dirty udders and concomitant bacterial infections, but also to facilitate milking for farmers. After the recent demonstration that this practice could be stopped without significant negative effects on the cleanliness of the cows' udder, the incidence of leptospirosis, or even on milk quality, tail docking is now prohibited in many countries, and has been renounced in France for many years.

The case of tail docking in horses. Tail docking was also traditionally performed on some breeds of horses to facilitate heavy work. This practice is almost no longer observed, mainly due to regulatory incentives (banned in competition). In the rare cases where it is still authorised (Trait du Nord horses used for agricultural or forestry work), veterinary surgeons deal with pain management (aseptic surgery on restrained animals with the use of analgesic drugs) for the procedure.

The case of tail docking in piglets. Tail docking of piglets cannot be discontinued as long as slatted floors remain in use in the majority of post-weaning and fattening units of piggeries because the risk of tail biting and cannibalism would be too high. Tail biting is a behavioural disorder of multifactorial origin with nutritional, environmental and genetic components. Environmental enrichment, including the provision of litter, prevention of nutritional deficiencies, maintenance of stable groups, and possibly genetic selection, would reduce the risk of the development of behavioural disorders and ultimately permit the practice of tail docking to be discontinued. This mutilation is not performed on organic farms where animals are reared on deep litter.

Beak mutilation in poultry. In some European countries (Sweden, Norway, the Netherlands), national law prohibits de-beaking and beak trimming. However, an exemption has recently been issued for the second time in the Netherlands. In fact, compliance with this regulation is currently only feasible in very specific situations, at the risk of facing an outbreak of feather pecking and cannibalism, and only for certain genotypes, such as the white egg-laying Leghorn hens. Giving up beak trimming is therefore not conceivable at the moment in France, where consumers buy mostly brown eggs.

Genetic selection as an option. Genetic selection is a long-term undertaking but it has the advantage of focusing on the causes rather than compensating for the consequences of pain-causing factors. The goal is to modify certain characteristics of the animal, either to accompany the phasing out of painful mutilations or to reduce the incidence of painful conditions/disorders.

Taking the example in dairy cows, selection was initially based on the quantity of milk produced. There was a rapid evolution in the selection criteria to improve the amount of dry matter in the milk and then to focus on morphology. Taking functional traits into consideration led to new indices on fertility, mastitis resistance and longevity, and finally, on ease of calving. The relative weighting of these criteria varies according to the breed. In theory, the aim in using these selection indices is to have a zero effect on fertility and a positive effect on all the other characters. The selection index for mastitis is based on the cell count in milk and, since recently, includes the incidence of clinical mastitis.

The genetic approach can also help eliminate certain sources of pain by intervening directly on the sources. Examples of positive developments on the limitation of pain are: a decrease in the incidence of the acute stress syndrome in pigs, for which the molecular mechanisms have been identified; ease of calving in cattle (for calving difficulties due to calf size); reducing unpleasant odours in meat without resorting to surgical castration.

Recent work on the behavioural characteristics of animals has shown that it is possible to develop selection programmes based on specific behavioural traits and therefore eliminate extreme phenotypes that cause problems. Several studies indicate that certain behaviours such as feather pecking in poultry, sometimes associated with cannibalism, or aggressive behaviour in mixed groups of pigs may be reduced or eliminated through the use of appropriate genetic selection programmes. Although research suggests that behavioural traits of animals are genetically controlled, detailed quantification remains difficult, so at the moment there are no plans for the large scale introduction of behavioural indices in breeding programmes.

One alternative to dehorning cattle may lie in the introduction of the 'polled' gene from polled breeds into horned breeds, especially in beef herds. This option is already being put into practice, especially in Britain, but it may generate cultural reticence since the horns are popularly considered emblematic of the species.

\subsection{2. 'Substitute': what possible improvements can be made to reduce the pain induced by certain farming practices?}

When a source of pain cannot be avoided, the next attempt is to improve the farming practices concerned in order to limit the intensity or duration of the pain. In general, training 
the personnel involved in livestock production always fosters progress. To ensure that improvements are put into practice, consideration needs to be given to providing incentives, such as regulations, communication, recognition of rearing schemes that go beyond the legal requirements or product promotion.

Alternatives for reducing pain associated with castration of calves. Castration of calves without anaesthesia is considered to be painful, regardless of the technique used and/ or the age of the animal (see pages 319-332). It seems, however, that castration with the use of Burdizzo forceps may cause less pain, in both intensity and duration, than surgical castration. In addition, based on the literature, it seems that calves should be castrated as early as possible, preferably at one week of age, as it seems to be much less painful than at 3 weeks of age, and even less than at 45 days. Leaving castration until 6 to 16 months of age, which is sometimes recommended to take advantage of the growth potential of intact males, makes the procedure more difficult to carry out and potentially more painful.

The case of dehorning cattle. Dehorning either calves or adult cattle without anaesthesia or analgesia is recognised as being painful. A recent study has given a lead for the improvement of dehorning techniques so as they are less painful by resorting to cauterisation of the horn-producing zones at a young age (before the age of 1 month which is before the start of horn development). It should be noted that this preventive method is therefore applied to all the animals and does not just target the animals causing problems.

The case of beak mutilations in poultry. Prior to finding long-term solutions, based on improved husbandry and breeding, to eliminate the source of the problem that currently necessitates beak mutilations, one feasible option to reduce the associated pain is to de-beak or beak trim chicks at a very early stage (see pages 319-332). Studies conducted on various species show that specific practical improvements should be considered for each farming system (genotype, species, mode of production).

Acute pain in infancy? Young age is still used as a justification for ignoring the existence of pain in animals. However, not only has it been clearly demonstrated that animals as well as humans can experience pain at a very early age, but also that such pain may alter their neurological development with the risk that they may be rendered more sensitive to pain in the long term. Most often, surgical procedures are performed in young animals for practical (small size, ease of restraint), medical (lower surgical risk, rapid healing) and cultural reasons. Pain treatment should be considered for two reasons: because the intervention is considered as potentially painful, and to avoid the risk of disturbing the development of the animal's nociceptive system with long-term consequences (sensitisation to pain, chronic pain). Such confusion between young age and sensitivity to pain is found in national regulations: dehorning cattle is recommended before 4 weeks of age, and castration of piglets within 7 days, without the requirement for any means of pain relief. The regulations are often misinterpreted as implying that the constraints of anaesthesia or analgesia can be dispensed with for surgical procedures conducted in infancy. While the regulations advocating that certain mutilations be carried out at a young age may be well-founded, the use of anaesthetic and pain relief ought to be recommended in accordance with the level of pain associated with the procedure. ('Relieve' pain - see 5.3.).

Pain related to chronic diseases. Many diseases are painful, particularly because of the development of inflammation, so disease control is important to ensure farm animal welfare. Even though pain due to chronic disease sets in progressively, giving animals an opportunity for adaptation, it often continues over a long period of time with long-term effects on animal welfare. Mutilations can induce chronic pain through neuromas, dental abscesses, etc.

In pigs. Lameness and foot disorders in pigs (especially osteochondritis) have multiple origins. The factors responsible for the development of these sources of pain are in particular linked to nutrition, housing, lack of exercise and genetic traits. Treating these disorders using pharmacological means or by limited adjustment to the housing remains difficult and success is limited to mild cases. The best solution lies in preventing and reducing the occurrence of these chronic disorders. Several measures can be taken to minimise injuries: improving the quality of the flooring (avoiding slippery floors and surfaces that are too hard or abrasive), favouring exercise (giving the animals the space and freedom to move, enriching their environment), or limiting aggressive behaviour (giving them sufficient space, adjusting the pens to reduce inter-individual competition and promote avoidance behaviours in social conflicts). Genetic selection against osteochondrosis may also reduce locomotor problems.

In cattle. Increasing the comfort of housing for animals is often crucial in preventing the occurrence of lameness related to foot or joint disorders. Attention should be paid to bedding quality, ensuring access to a resting area, ease of access to sufficient food and water, providing adequate facilities for the access to the milking room, etc.

In poultry. Musculoskeletal disorders (lameness) are still found in poultry, but their prevalence is now reduced because of efforts made by farmers and breeders. They are most often of multifactorial origin with a strong genetic component and a combination of nutritional and husbandry factors contributing to their appearance. It has been established that the occurrence of diseases associated with these disorders is usually increased by nutritional growth stimulants. 
Several basic options should be considered to prevent and therefore reduce pain associated with musculoskeletal disorders:

- Decreasing food availability which reduces the onset of growth. In order to maintain high BW at slaughter, it has been recommended to alternate a day of high-energy diet with a day of high protein diet.

- Using lighting programmes which provide longer nights in the first weeks of life and which slows down the onset of growth.

- Promoting physical activity for young animals which strengthens the musculoskeletal apparatus, for example by increasing the distance between feeders and drinkers.

It has also been shown in laying hens that bone quality is a moderately inheritable trait; therefore genetic selection on this trait may be effective in reducing the number of bone fractures due to osteoporosis.

Organic poultry and other alternative production systems that emphasise access to outdoor areas in their code of practice may have mixed effects in terms of limiting pain. Mortality rates are slightly lower ( $1 \%$ to $2 \%$ ) in slow growing genotypes of broilers compared with conventional production systems even though they are reared over a longer period of time. In contrast, the mortality rate in laying hens that have access to an outdoor area is about twice that of caged animals. This is due to specific diseases and increased pecking behaviour. Pecking and mortality rates are currently being reduced by selecting genotypes better suited to outdoor conditions. Meanwhile, a high incidence of fractures of the sternum has been reported in indoor systems using above-ground platforms with several levels (aviaries), resulting in all likelihood from the birds impacting against the edge of the platforms when they try to fly.

In horses. Painful diseases relating to farming conditions have never been reported in horses. An interesting point is the difference in the way horses for meat production are treated in comparison to those reared as pets or for sport (see Section 5.3.3.).

\subsubsection{Improvement of slaughter conditions}

The improvement of facilities and slaughterhouses. Improving structures and procedures in slaughterhouses is strongly recommended, such as:

- Designing loading and unloading platforms, stalls, yards and corridors to facilitate unloading the animals into lairage with minimal use of electric goads or sticks, which inflict pain.

- Properly equipping abattoirs to facilitate the flow of animals. The requirements include avoiding visual obstacles or contrasts in lighting, and installing equipment such as treadmills, anti-backup devices, etc.

- Ensuring stunning boxes restrain the animals properly but not too tightly. Ensuring good maintenance of the stunning devices.
Other parameters seem crucial for minimising animal pain at the slaughterhouse and for improving working conditions for staff, especially in terms of safety:

- The right balance between the production rates demanded and the realistic potential given the facilities in the abattoir.

- Appropriate training of the personnel.

\section{Improving the stunning procedure}

In standard slaughter procedures. Recovery of consciousness before the animals are bled to death means that they may feel pain again. Recovery of consciousness varies with the species and the stunning method used but the risk of recovering may be limited by:

1. Adapting the procedure. For electrical stunning, the voltage and amperage required to induce instant insensibility must be adapted to the species. The electrodes must be held in firm contact with the head and the equipment correctly set up. For captive bolt, the device must be properly positioned (generally on the forehead) and adapted to each species. For gas, proper concentration and duration of exposure must be ensured for all the animals, for example by limiting the number of animals (especially poultry) exposed to gas at the same time, so as a sufficient amount of gas rapidly reaches all the animals and in the case of reversible stunning, there must be enough time to bleed all the animals before they have a chance of recovering.

2. Reducing the interval between stunning and bleeding to limit the risk of recovery.

3. Increasing the duration of unconsciousness, for example through the application of electrical head-body stunning (instead of head only stunning) which generally induces deeper electronarcosis with longer loss of sensibility (often resulting in the animal's death).

In ritual slaughter procedures. In France, ritual slaughter by throat cutting is often carried out without stunning the animals either before or after bleeding. The manner in which the act is carried out is crucial to its effectiveness in establishing a rapid loss of consciousness. As with standard slaughter procedures, the training of religious slaughterers and slaughterhouse operators to ensure consistent and effective ritual cutting, the equipment used, the method of restraining the animals and an adapted/appropriate work rate are all factors determining success. If no conventional pre-cut stunning is applied, there are two conceivable options for avoiding the pain associated with ineffectual bleeding and delay in loss of consciousness, which is often observed in cattle.

(1) In some countries (Britain, the Netherlands and New Zealand and for species such as poultry, sheep and goats) reversible stunning is performed before Halal sacrifice. Further research is needed to ensure that the animals subjected to reversible stunning do not perceive pain in the time lapse until death through bleeding (Box 2).

(2) Stunning the animals after ritual bleeding, as practised in Austria for Halal or Shechita meat. Consideration can be given to routine stunning of all animals after throat 
cutting or just of cattle when impaired bleeding due to the formation of blood clots at the caudal ends of the carotids retards the loss of consciousness. Thus, the rapid use of a captive bolt just after bleeding for animals that bleed too slowly and do not show satisfactory loss of consciousness within seconds, would shorten the pain and discomfort of slaughter. One difficulty here is in the rapid recognition of animals that bleed too slowly in order to decide on the use of stunning.

There is currently very little data concerning on-farm killing (slaughter performed by the farmer without consumption of carcasses) for emergency reasons relating to the welfare of the animal, routine culling of surplus newborn and unwanted, low value animals (e.g. very weak 1-day-old rabbit pups or chicks) and non-emergency slaughter of casualty animals or for the purposes of disease control. The regulations are more precise in the case of sanitary culling. Weak or injured animals that are unfit for transport to the abattoir must be slaughtered on farm but the terms for on-farm methods of euthanasia are not clearly defined. Administration of barbiturates or overdoses of anaesthetic by veterinary surgeons is rarely adapted to these situations, mainly because of the cost.

\section{Box 2 Ritual slaughter in New Zealand}

Personal communication from D.J. Mellor, Professor of Animal Welfare, Applied Physiology and Bioethics, co-director of the centre of animal welfare and bioethics

For at least 30 years, in New Zealand, commercial slaughter of lambs/ sheep for export has been by the Halal method, including head-only, reversible electrical stunning. The Muslim requirements as expressed then, and reaffirmed since, of the animal being alive and healthy, and that death be caused by the neck cut, are met by the prior use of this form of stunning, because it does not itself kill the animal. When this was demonstrated to Muslim clerics, and provided that the neck cut is done by a Muslim slaughterman well versed in the religious requirements, they accepted this form of commercial slaughter as complying with Halal requirements. This approach remains in place today. Of course, this approach also meets the general animal welfare requirement in commercial slaughterhouses that the neck cut must be preceded by effective stunning

For the New Zealand Jewish community the question of preslaughter stunning has been problematical, because it has been regarded as against Kosher slaughter requirements. For the last 10 years no sheep or cattle have been slaughtered by the Shechita method in New Zealand, as the Jewish community here has chosen to import their requirements for such meat while a new code of welfare for commercially slaughtered animals was being developed.

Comparatively small numbers of chickens have been slaughtered via the Shechita method for use only within New Zealand by the local Jewish community. This matter is still under review, and requires consideration to be given both to the New Zealand Bill of Rights Act, which allows the free practice of religion and belief, and the Animal Welfare Act, which prohibits the slaughter of animals in ways that cause unreasonable and unnecessary pain and distress, but at the same time states that the New Zealand Bill of Rights Act provisions for free practice of religion and belief must also be considered. This matter remains under review
Council Directive 93/119/EC of 22 December 1993 on the protection of animals at the time of slaughter or killing, fixed minimum common rules in the European Community. The application of these rules is, however, little controlled on-farm and can potentially lead to painful killing practices (choking, stunning). In rabbits, the killing of the weakest newborns from each litter remains without regulation despite a significant number of individuals concerned (around $6 \%$ to $8 \%$ of births).

The need to establish detailed standard operating procedures that are adapted to each farm condition and each species is highlighted in the literature. For small animals $(<3 \mathrm{~kg})$ maceration, using a large high-speed grinder, or gassing are the most commonly used techniques because they cause rapid death.

For larger animals, using proper stunning or sedating methods before killing them in a rapid and standardised manner, and the verification that the animals are actually dead, are recommended. The problem of the availability to breeders of slaughter equipment and euthanising drugs remains a significant obstacle. A solution based on the possibility for farmers to use euthanasia services at a lesser cost, managed by a group of technicians trained to perform animal euthanasia, would reduce pain due to non-conforming killing methods.

\section{3. 'Soothe': pharmacological treatment of pain}

\subsubsection{General principles of pain management in veterinary medicine}

Pain management methods have been developed for use in veterinary medicine and in theory are suitable for livestock. Therapeutic treatments for birds and fish are less available because of physiological differences and the low number of studies in these species. In practice, the use of analgesics and veterinary treatments for animals reared for food production is limited because of certain restraints (limited pharmaceutical market, limiting the risk of drug residues in meat, availability to farmers). As such, pharmacological treatments are given as a last resort, and the priority is rather focused on prevention (see 5.2.). When the sources of pain cannot be avoided (suppress) or reduced (substitute), the possibility of relieving pain with a veterinary treatment (soothe) becomes an ethical issue.

General recommendations. Analgesia is based on three good practices: (i) preventive analgesia (if possible, prevent pain in any animal undergoing mutilation), (ii) interventional analgesia (always treat pain during surgery); (iii) rescue analgesia (or curative) which aims at relieving any pain so as to limit the symptoms and the economic impact. The analysis of a painful situation allows an appropriate analgesic procedure to be established (see Table 2).

Local anaesthesia. When possible, local or regional anaesthesia is fast, easy and inexpensive to perform. There are various ways to administer lidocaine as close as possible to the affected nerves (cranial nerves, nerves innervating the 
Levionnois and Mormède

Table 2 Questions to be posed in choosing an analgesic strategy

\begin{tabular}{llll}
\hline \hline & Questions to be posed & Procedure chosen & Substance (administration) \\
\hline 1 & Possibility of local anaesthesia? & Local anaesthesia & Lidocaine (perineural) \\
2 & Inflammatory process? & Systemic analgesia & NSAIDs (IV, PO) \\
3 & Chronic pain or severe and persistent pain? & In association with: & $\begin{array}{l}\text { Ketamin (SC) } \\
\text { Butorphanol (IV) } \\
\end{array}$ \\
& $\S$ & Lidocaine (IV) \\
& & $\begin{array}{l}\text { Xylazine (peridural) } \\
\text { Xylazine or detomidine (IV) }\end{array}$ \\
& Need to restrain? & Sedation & Ketamine (IV), Isoflurane (Inhalation) \\
\hline
\end{tabular}

*NSAIDs = non-steroidal anti-inflammatory drugs, IV = intravenous; PO = per Os (oral); SC = sub-cutaneous.

Systemic: SC or PO.

${ }^{\$} \mathrm{~A}$ Marketing Authorization (MA) has not been issued for lidocaine and butorphanol for intravenous injection in cattle and therefore they are subject to the provisions of the cascade for minimum withdrawal periods (see 5.3.2)

testis and the limbs, epidural, paravertebral). This anaesthesia permits the short-term reduction of pain (a few hours maximum). Complementary analgesic treatment is usually required for longer duration of action to relieve post-surgical pain, and in particular pain of inflammatory origin.

Several studies indicate that regional anaesthesia is effective in desensitising the testis and the spermatic cord when performed before castration in horses, cattle, small ruminants and pigs. The anatomy of birds does not favour the use of local anaesthesia even though it has been considered for the castration of broilers. Other painful conditions can be relieved by local anaesthesia, such as dehorning calves (cornual nerve anaesthesia) or inserting nose-rings in bulls (anaesthesia of the infraorbital nerve).

Anti-inflammatory-based analgesia. The administration of non-steroidal anti-inflammatory drugs (NSAIDs) is recommended for surgical or accidental tissue trauma to limit the development of inflammation and pain associated with it. Inflammation starts shortly after surgery and attenuates over the following days.

Note that for the treatment of chronic pain, whether severe or persistent, the most effective analgesics are morphine derivatives (opioids) and are commonly used in pets.

The use of pharmacological products for restraint: sedation or general anaesthesia. Finally, the possibility of resorting to pharmacological products for animal restraint should be mentioned. When physical restraint and pain management using local anaesthetics and anti-inflammatory drugs are not possible or are inadequate, two types of pharmacological agents can be administered. Sedatives (Xylazine or detomidine) can induce a deep state of detachment and relaxation enabling immobilisation of the animal in standing position, the effectiveness of which depends on the intensity of pain. General anaesthesia is more efficient and induces limited and reversible complete loss of consciousness required for certain surgical procedures (deep laparotomy, orthopedic surgery, major injuries). General anaesthesia is more difficult to use routinely on-farm and in general can only be practised by a veterinary surgeon. The special case of the use of general anaesthesia by farmers for the on-farm castration of piglets will be developed in a separate section.

\subsubsection{Limitations on the use of pharmacological agents on livestock}

A basic rule for consumer protection is to reduce the risk of drug residues in food (antibiotics, anti-inflammatories, toxic or active substances). Thus, EU regulation provides a list of active substances for which a maximum residue limit (MRL) is set. Only these products can be administered to livestock. In addition, the MA for a pharmaceutical drug includes a withdrawal period during which animal products from an individual that has received a pharmacological treatment are not suitable for human consumption. This withdrawal period is based on experimental and clinical studies. During this period, milk or eggs are discarded, and the animal cannot be slaughtered for human consumption. Veterinary surgeons are responsible for ensuring that withdrawal periods are complied with. Thus, sedatives and anaesthetics granted full MA are available for several species of livestock and can be used if needed.

When a drug has not been granted MA for a certain indication in a given species, such as for anti-inflammatory use, the 'cascade' provision can apply for the prescription of a product for which the MRL has been set, and for which the MA has the same indication in a similar species, or in this species but for another indication. In such case, a minimum withdrawal period is implemented (e.g. in cattle, 28 days for meat and 14 days for milk). This system allows, for example, the use of Lidocaïne in cattle. Prescribing an anti-inflammatory drug for systematic peri-surgical administration by the farmer is therefore possible, provided that the farmer is also informed on the withdrawal period. The development of specialty pharmacological agents by pharmaceutical companies in view of obtaining MA in Europe, with indications for use in analgesia in farmed species, is a very expensive process. Legislative change is required to make the analgesic market more attractive. In the meantime, one option may be to facilitate the possession and use of such veterinary products in on-farm pharmacy.

Lidocaine is a complex example because it combines recourse to the 'cascade' principle (no MA for livestock in France) 
and the intervention of a practitioner. Like the use of sedatives and general anaesthetics, performing a local anaesthesia is regulated by the Rural Code in Section IV. Practicing veterinary medicine and surgery on animals is restricted to veterinary surgeons. While it is possible for farmers to have prescriptions for local anaesthetics filled, anaesthetising a nerve by subcutaneous injection of the product is reserved for veterinary surgeons. A special exemption is granted nonveterinary surgeons by law (article L243-2 of the Rural Code) for surgical castration in species other than horses, dogs and cats. This opens the possibility of considering whether local anaesthesia could be performed by the farmer on the condition that it is recognised as being part of the intervention. This would require special mention in the Rural Code. In the case of dehorning, giving farmers permission to carry out the procedure when the calves are $<4$ weeks of age, but in the absence of any form of analgesia, and prohibiting them from anaesthetising the cornual nerve (which is considered as an act of veterinary medicine) does little to foster the use of this simple and inexpensive pain relieving procedure.

For farmers to be permitted to administer local anaesthetic in combination with NSAIDs, veterinary surgeons must ensure they are adequately trained. They must also accept responsibility for the consequences of the delegation and for the use of drugs without an MA. This is currently the case in Switzerland, for example, for gas anaesthesia for castration of piglets. In this country, the veterinary services train field veterinary surgeons, who in their turn train farmers in anaesthetising their animals, and in the cleaning and the maintenance of the equipment.

Another obstacle to the use of analgesics or anaesthetics is the persisting lack of awareness among farmers and veterinary surgeons of the problem of pain. An information campaign among farmers would not only lead to better identification and recognition of pain, it would more importantly encourage the use of preventive and curative practices. The cost of administering anaesthetic and analgesic drugs also appears to be a major disincentive for farmers. Yet, the cost of the anaesthetic itself is often low ( $\sim 1$ euro for the amount of lidocaine necessary to anaesthetise the cornual nerve). Indirect costs (work time) seem to be an even more important economical constraint to farmers who do not see in pain management a direct return on their investment. An economic study undertaken in 2009 focused on the additional costs generated by surgical castration with anaesthesia in pigs. For a production of identical quality, the increase in cost of meat production is estimated at $0.1 \%$ to $0.3 \%$ in the case of local anaesthesia administered by farmers, and $0.9 \%$ to $1.6 \%$ for local or general anaesthesia administered by a veterinary surgeon. In the latter case a large part of the variation was due to the size of piggery, the cost being more significant for small units. Moreover, even if French or European authorities consider that the presence of drug residues in very low concentrations is safe, export to other countries could be penalised if those countries contest the notion of there being no risk. The use of veterinary products must always be conducted with openness, with checking of doses and adherence to withdrawal periods so as to maintain control over the impact on food.

From a consumer's perspective, the development of a product in observance of practices guaranteeing animal welfare at the risk of increasing the presence of drug residues, must maintain a substantial equivalence in the animal product (like-product) and not lead to a degradation of its nutritional, health and gustatory qualities.

\subsubsection{Examples of pain management applicable to livestock}

Pain management for mutilations: the example of castration. Dealing with pain during castration is particularly difficult because castration is a procedure that farmers are entitled to perform while access to painkillers and anaesthetics is restricted to veterinary surgeons. This situation gives justification to the long-standing practice of performing this act in the absence of analgesia. Nonetheless, farmers could be authorised to administer local or general anaesthesia and NSAIDs in a well-defined framework once these acts are considered an integral part of the procedure.

One technique of general anaesthesia seems promising for the castration of piglets: applying a mask for the inhalation of anaesthetics (isoflurane with or without $\mathrm{N}_{2} \mathrm{O}$ ) in oxygen. This technique is easy, quick and without post-operative complications. It is performed by farmers in Switzerland but its use is not without drawbacks (release of a toxic gas into to the environment). A variation based on the administration of carbon dioxide is used in the Netherlands. Administering local anaesthesia, sometimes in association with an anti-inflammatory drug, as is practised in Norway, is another possibility.

In broiler chickens, a variety of available substances have been tested for conducting caponisation under general anaesthesia and/or local analgesia, but the implementation of this method is difficult because these products have a very heterogeneous duration of action in birds. This heterogeneity of effect can lead to cannibalism during the postoperative period if the first animals to recover consciousness are not immediately removed from their conspecifics. Gas anaesthesia is used in veterinary practice for pets or wild birds and also in experiments but has not been tested in poultry farms for technical and financial reasons.

In ruminants, surgical castration combined with chemical restraint (sedation or general anaesthesia), local anaesthesia (in the distal pole of each testis) and analgesia (if possible anti-inflammatory drug $20 \mathrm{~min}$ before surgery to relieve postoperative pain), is practiced in some countries and is recommended in organic farming. Adopting these practices is conceivable but the increased cost would be significant.

In the case that use of analgesia for conducting mutilations becomes widespread, and more particularly in regards to the specifications for organic farming, it should be noted that pain and anxiety are factors that destabilise homeostasis and lead to reduced immunity. The administration of analgesics such as anti-inflammatory drugs also depresses the immune system, but so far there has been no clinical study examining the balance of the benefits brought about by administering in pain relief in terms of homeostasis and 
the negative effects of anti-inflammatory drug administration on immunity depression. However, it has been established that the physiological effects of untreated pain are related to its duration and intensity just as the side effects of anti-inflammatory drugs are dependent on the dose and the duration of the treatment. Therefore, once again, it appears that the strategies aimed at eliminating or substituting the sources of pain prove to be more effective than those aimed at relieving pain afterwards. For pain management during surgical castration or castration by the use of a rubber ring, for example, local anaesthesia is, according to our current state of knowledge, the most effective and easy to use and is followed by fewer side effects. The administration of antiinflammatory drugs is also possible. The dose and duration of treatment need to be adjusted to the type of pain caused. Preventive administration before the mutilation probably gives the most effective outcome and enables the shortest treatment time. A single administration minimises side effects while providing a minimum level of analgesia.

Managing pain caused by diseases. Certain diseases, mostly chronic diseases but also those involving a significant inflammatory component, may be accompanied by clinical signs of moderate to severe pain that require pain management. NSAIDs can relieve pain over several days and have proven to be effective for the treatment of lameness. For clinical mastitis, NSAIDs are associated with local anaesthesia in the case of teat infection, for example, or butorphanol or xylazine in case of severe visceral pain. The systemic administration of anti-inflammatory drugs is often necessary to reduce the symptoms of acute mastitis and to avoid the development of toxic mastitis. In contrast, the use of intramammary corticosteroids is controversial and should be assessed on a case by case basis to allow a rapid improvement in local symptoms.

Pain management in the horse industry. The horse industry benefits from modifications to the regulations specific to horses. Resulting from the flexibility required for veterinary treatments used for horses in sport and recreation, the regulations for meat horses allow an additional list of veterinary pharmaceutical substances (denoted 'essential') which comprises several anaesthetics and analgesics for which the use is permitted despite the fact that no MRL has been established. A withdrawal period of 6 months is imposed before slaughter. While this model could be followed for animals from which only the carcass enters the human food chain, a withholding period of 6 months appears difficult to put into place to allow the systematic use of some products in the cattle industry or in other industries. Further research is required to propose minimum standard withdrawal periods in the dairy or the poultry industries.

\subsection{Summary}

In the current state of knowledge and according to the rearing and slaughtering situations, there is flexibility available for limiting or avoiding pain by adapting equipment, techniques or practices in place on farms and in slaughterhouses.

Medical treatments for pain are available, but current regulations, which favour limiting the risk of drug residues in animal products, and cost and labour optimisation are often barriers to their use.

Some strategies for genetic selection and/or certain rearing codes of practice may reduce the risk of the development of pain.

The introduction of incentives and enforcement of adequate regulations can facilitate the use of solutions to avoid, reduce or treat pain in farm animals (see legislation, training and information for industry personnel).

In some species such as poultry and fish, there are few analgesic procedures available. Their implementation or development would require experimentation and validation on a commercial scale. 